\title{
An investigation of everyday prospective memory
}

\author{
RICHARD L. MARSH, JASON L. HICKS, and JOSHUA D. LANDAU \\ University of Georgia, Athens, Georgia
}

\begin{abstract}
Prospective memory, remembering to carry out one's planned activities, was investigated using a naturalistic paradigm. Three experiments, with a total of 405 participants, were conducted. The goal was to demonstrate that the cognitive processing underlying successful everyday prospective remembering involves components other than mere "memory." Those components are probably best represented as individual differences in various cognitive capacities. More specifically, metamemory, attentional capacities, and planning processes that reprioritize intentions according to the demands of everyday life may determine how people actually accomplish the plans they establish for themselves. The results of these experiments suggest that researchers interested in the topic will have to contend with a multidimensional set of factors before any comprehensive understanding of prospective remembering can be realized.
\end{abstract}

This article concerns the attentional and memory systems that people use to guide and control their behavior. Loosely defined, research on prospective memory investigates how people establish their future goals at one time and then, at later times, attempt to fulfill those delayed intentions. Prospective memory is often contrasted with retrospective memory, which is memory for what one has done or encountered in the past (e.g., Einstein \& McDaniel, 1990). Adopting the perspective that prospective and retrospective memory are somehow qualitatively different phenomena has allowed researchers to discover many interesting similarities between the two types of memory. For example, prospective memory is better when (1) the retention interval is shorter between forming an intention and the time it is to be completed (Loftus, 1971), (2) incentives are offered to promote remembering (Meacham $\&$ Singer, 1977), or (3) the prospective tasks are more important (Kvavilashvili, 1987, 1992). Other studies, however, have examined factors that are unique to prospective memory, such as how comfortable the individual feels about completing the intention (Meacham \& Kushner, 1980 ), the various strategies used to remember to perform an action in the future (Harris, 1980), how prospective memory skills might develop from childhood (Beal, 1988), whether age-related differences exist (Craik, 1986;

\footnotetext{
This research was supported in part by a grant from The University of Georgia Research Foundation, Inc. and by Sigma Xi Grants-in-Aid. Portions of this work were presented at the Prospective Memory in Older Adults Workshop sponsored by the Southeastern Center on Applied Aging, August 1995. Appreciation is expressed to Caren M. Jones for helpful comments offered concerning an earlier version, as well as to Geoff Loftus and Jonathan Schooler. The second author served as the primary experimenter in all of the experiments, and the results of the first experiment constituted his master's thesis. Correspondence should be addressed to R. L. Marsh, Department of Psychology, University of Georgia, Athens, GA 30602-3013 (e-mail: marsh@meme.psy. uga.edu).
}

—Accepted by previous editor, Geoffrey R. Loftus
Einstein, McDaniel, Richardson, Guynn, \& Cunfer, 1995), and how psychologists fulfill their intentions (Park, Smith, \& Cavanaugh, 1990).

According to one view, prospective memory is supported by a set of human planning processes. In accord with this conceptualization, recent theoretical frameworks have placed prospective memory squarely at the cognitive crossroads of memory, attention, and action (Ellis, 1996). Thus, studying other cognitive components involved in completing one's planned activities besides "memory" should advance our understanding of this complex phenomenon. This article reports three attempts to investigate several other nonmemorial components of prospective remembering using a very different paradigm from other studies found in the literature.

Previous paradigms examining prospective memory can be broadly classified as naturalistic or laboratorybased. In the naturalistic studies, people were asked to mail postcards to the experimenter (Meacham \& Singer, 1977), to press a button on a clock several times a day (Wilkins \& Baddeley, 1978), or to make phone calls at prespecified times during a day or week (Maylor, 1990; Moscovitch, 1982). The major criticism with these earlier studies was a lack of experimental control. For example, prospective tasks, such as returning postcards or phoning the experimenter at preset times, are troublesome because the experimenter does not know what memory aids, if any, are used by the participants in order to comply with the demands of the experiment. Moreover, the intention is really not the participant's, but rather, the experimenter's. In contrast, there has been recent interest in returning to laboratory-based investigations of prospective memory. Einstein, McDaniel, and their colleagues (Einstein \& McDaniel, 1990, 1996; Einstein et al., 1995) have pioneered two computer-based tasks, one event-based and the other time-based.

In the time-based task, people monitor a clock and respond at fixed intervals (e.g., every $10 \mathrm{~min}$ ) while performing a second, attention-demanding task (an ongoing 
short-term recall task akin to the Brown-Peterson task). Because of the difficult nature of this retrospective memory task, the prospective task is believed to be "subordinate" and thereby to approximate real-life prospective remembering when people are busily engaged in other activities. The dependent measures in this paradigm include the number of successful responses and the frequency of clock checking. In the event-based task, participants are given one or two words to remember (e.g., rake) and are told to press a key whenever they encounter the word(s) in the ongoing recall task. Both of these laboratory-based tasks represent new attempts to collect empirical data on prospective remembering. Moreover, both paradigms acknowledge that remembering to carry out a task in the future inherently involves a self-initiated cuing process akin to reminding oneself. Whether more self-initiated processing is required by the time-based task as compared with the event-based task is still debated (Craik, 1986; Einstein et al., 1995). Regardless, our approach in this article was to examine the likely correlates of this selfinitiated processing that is used outside of the laboratory by studying the effectiveness of such things as the use of a daily planner. Intuitively, successful remembering of future engagements might require that one periodically refresh the plans and goals set forth for that day or week, and that includes remembering to check a daily planner if one is kept.

Assuming that prospective memory performance in the laboratory is often poorly correlated with performance outside the laboratory (e.g., Mayhorn, Park, Morrell, \& Marsh, 1995), our approach was to freely admit that sacrificing some laboratory control in favor of ecological validity might nevertheless yield important insights regarding how people remember to accomplish their intentions. As our starting point, we assumed that cognitive capacities other than retrospective memory were critical to prospective performance (Dobbs \& Reeves, 1996). That is, accomplishing one's intentions involves planning, monitoring, and prioritizing processes that could be largely driven by attentional and inhibitory mechanisms. For example, changes in focused attention and attentional switching capacities afford people the opportunity to engage in temporal monitoring processes and to initiate self-cued remindings about upcoming planned activities. These capacities also allow people to review and to revise the activities that they have planned on the basis of new goals, new events, and new contingencies that occur in the course of everyday cognition. Rather than directly manipulating levels of attention (see Marsh \& Hicks, 1998) and the strategies that people use to remember their intentions, the paradigm reported here took participants as they came to the laboratory, with all of their variability, individual differences in attentional capacities, and strategies for completing their intentions. The basic paradigm required participants to come in on one day to document the activities that they had planned for the coming week. One week later, they returned and documented what they had actually ac- complished, providing reasons for failures to complete their intentions. Across the three experiments, how people remembered their plans was manipulated or various capacity measures were collected, such as metrics of sustained attention, attention switching, retrospective memory, and metamemory.

How might this approach to studying prospective memory enhance our understanding of the phenomena? Chiefly, the approach examines multiple, simultaneous intentions that people must juggle over the course of a week. Hitherto, most laboratory studies have investigated a single intention that is performed once (e.g., Dobbs \& Rule, 1987; Loftus, 1971) or the same intention performed repeatedly (e.g., Einstein \& McDaniel, 1990; Wilkins \& Baddeley, 1978). Second, the paradigm investigates intentions that participants established for themselves, and not intentions dictated by the experimenter (cf. Ellis \& Nimmo-Smith, 1993). Third, the approach begins to address the planning and the reprioritization processes that people use over extended periods of time that are impossible to study with the brief establishment of an experimenter's intention supplied to participants in a laboratory setting. That is, the paradigm acknowledges that, from the time of formation, most intentions are delayed for some extended period (usually because they cannot be accomplished immediately) and that these intentions are then executed as environmental and cognitive demands permit. Fourth, and in a related way, the approach investigates the outcome of the natural processes of updating, abandoning, and revising one's plans as they occur simultaneously with all the various (and typical) human difficulties that people have managing their time and behavior (see Rabbit, 1996, citing the need for such a paradigm). Fifth, and finally, the paradigm examines people's natural use of memory aids, such as daily planners, to determine how such variables may affect what they ultimately accomplish.

\section{EXPERIMENT 1}

Experiment 1 was conducted to assess how many plans people established for themselves over the course of a week and to determine what proportion was actually accomplished. Another goal was to understand the underlying reasons why some intentions went unfulfilled. A final goal was to determine whether individual differences in the use of memory aids, as well as native differences in attentional and memory capacities, were related to prospective memory performance outside the laboratory.

\section{Method}

Participants. One hundred thirty-five undergraduates from the University of Georgia volunteered and received partial course credit for their participation. The participants were tested in groups of 10-20 people. Each group was tested in two sessions conducted 1 week apart.

Materials. The basic materials consisted of three different performance assessments: (1) planning activity sheets measured peo- 
ple's success at completing their intentions, (2) general memory ability was measured both by self-report and by an objective retrospective recall measure, and (3) attentional capacity was measured by two objective tests and one self-report measure. The planning activity sheet was divided into six sections corresponding to the categories of plans most frequently cited by participants in a similar, but retrospective, task (Meacham \& Kushner, 1980). For brevity, these six categories are described in the Results section. The participants were given $10 \mathrm{~min}$ (which was ample time) to write down their plans for the coming week under each of the section headings. The form also asked several other questions regarding people's habitual use of a daily planner (i.e., calendar). People used this form again when they returned 1 week later to document completion (or noncompletion) of the activities they had intended that week.

The participant's general memory ability was assessed with three measures. Two of these were self-report metamemory questionnaires. One assessed people's beliefs about how good their memory was (Everyday Memory Questionnaire, hereafter the EMQ; Martin, 1986), and the other assessed how often they experienced failures in memory and action, such as forgetting other people's names or where they left a book for later use (Cognitive Failures Questionnaire, hereafter the CFQ; Broadbent, Cooper, Fitzgerald, \& Parkes, 1982). In addition to these two measures, the participants were given 24 low-frequency words to remember for an immediate free recall test.

To measure attentional capacity, three standard measures of attention were administered. The Symbol Digit Modalities Test (hereafter the SDMT) is a global assessment of attention that contains a measure of attentional switching capacity that was originally developed to screen for learning disabilities in people without physical brain abnormalities (Smith, 1968). In addition to this attentional switching assessment, the Ruff 2 and 7 Selective Attention Test (hereafter the $2 \& 7$ test) is known to measure one's capacity for sustained attention on a selective crossing-out task (Ruff, Niemann, Allen, Farrow, \& Wylie, 1986). The third measure, the Everyday Attention Questionnaire (hereafter the EAQ; Martin, 1986), is a selfreport of one's ability to carry out specific activities while simultaneously performing various other tasks (i.e., dual-task performance).

Procedure. During the first session, the participants received the planning activity sheets and were instructed to document the activities they had planned for the upcoming week. They were also asked to rate the importance of each plan on a 7-point Likert scale. Those who regularly used a daily planner were notified beforehand to bring it to the laboratory and were encouraged to consult it in order to document their plans. The participants took the retrospective memory test consisting of a randomized list of 24 low-frequency words. Each word was presented for $5 \mathrm{sec}$ on an overhead projector at the front of the room. The participants were immediately given $2.5 \mathrm{~min}$ to write down all of the words they could remember. This retrospective memory assessment and the recording of plans constituted the first session.

Upon their return 1 week later, the original planning activity sheets were returned. At this time, the participants recorded on a separate sheet whether each plan had been completed. If a plan was not completed, space was provided to explain why the activity was not fulfilled as originally intended. The EMQ, the CFQ, and the EAQ were administered next, in that order. Each questionnaire was self-paced with the restriction that the participants could work on only one questionnaire at a time and could not return to a previous measure after finishing it. After the self-report assessments, the SDMT and the 2 $\& 7$ test were administered to the entire group, with the experimenter pacing and calling out ending times as necessary.

\section{Results and Discussion}

The results concerning the participants' failures to fulfill their intentions (and the reasons they provided) are discussed first, followed by an analysis of the memory and attention measures. In this second analysis, particular differences are noted between those who use memory aids such as daily planners and those who do not. Of the 135 participants, $78(57.8 \%)$ reported that they normally used a daily planner and $57(42.2 \%)$ reported that they did not. Hereafter, we use the term recorders to refer to the participants who habitually used a daily planner or some other written list to keep track of their intentions and obligations; we use the term nonrecorders, for those who did not use any written device. When confidence intervals (CIs) are reported, they represent $95 \%$ intervals around sample means.

Unfulfilled intentions. Overall, the participants reported an average of $15.5(S D=5.34)$ plans for the upcoming week; that number did not differ for the recorders and the nonrecorders, with 15.5 and 15.4 plans for the week, respectively $(\mathrm{CI}=14.6-16.4)$. The classifications of plans on the planning activity sheets were (1) commitments and appointments (e.g., a dentist appointment), (2) intentions to commit (e.g., calling to establish an appointment), (3) intentions to complete (e.g., having to return something or to borrow something from a friend), (4) intentions to study (e.g., doing homework), (5) intentions to communicate (e.g., writing, telephoning, or sending a letter), and (6) miscellaneous intentions (e.g., taking medicine or feeding a friend's pet). Table 1 provides the percentage of plans in each category that were not completed, averaged across all participants.

Interestingly, the recorders and the nonrecorders had equivalent overall noncompletion rates (see CIs in the bottom two entries of column 1 of Table 1). Moreover, in standard significance tests, the two variables of plan category and status as a recorder versus a nonrecorder did not interact $[F(5,430)<1]$, indicating that noncompletion rates were not different by category according to whether or not the participants used a daily planner. This same analysis (due to linear dependence of the variables) also serves to confirm that the recorders and the nonrecorders did not have proportionately different plans across the six categories. Examination of the CIs in the first column of Table 1 clearly shows that noncompletion rates were very different depending on the category of plan. Greater rates of noncompletion appear localized to two categories that can be characterized as the participants' uncommitted intentions (i.e., intentions to communicate and intentions to complete). In contrast, previously established intentions, characterized by meeting appointments or doing assigned homework, showed markedly smaller noncompletion rates.

Given that there was no overall difference between those who used daily planners and those who did not, the various reasons that the participants gave for failing to complete their intentions were examined, pooled over that variable. In general, the participants gave four different classes of reasons for failing to complete their plans: (1) they completely forgot the intention, (2) they reprioritized their activities (e.g., decided something else was more important or more favorable to do, capitalized on better opportuni- 
Table 1

Percentages of Uncompleted Plans by Category

and Percentages of Reasons Given for Those Failed Intentions in Experiment 1

\begin{tabular}{|c|c|c|c|c|c|}
\hline \multirow[b]{2}{*}{ Plan Category } & \multirow{2}{*}{$\begin{array}{c}\text { Overall } \\
\text { Noncompletion }\end{array}$} & \multicolumn{4}{|c|}{ Reason Given for Noncompletion } \\
\hline & & Forgot & Reprioritized & Cancelled & Impossible \\
\hline Commitments and Appointments & $\begin{array}{c}16.5 \\
(12.3-20.7)\end{array}$ & 8.1 & 38.7 & 21.0 & 32.2 \\
\hline Intentions to Commit & $\begin{array}{c}29.5 \\
(23.3-35.7)\end{array}$ & 23.6 & 50.6 & 22.5 & 3.3 \\
\hline Intentions to Complete & $\begin{array}{c}36.3 \\
(29.7-42.9)\end{array}$ & 9.0 & 55.9 & 15.3 & 19.8 \\
\hline Intentions to Study & $\begin{array}{c}17.5 \\
(12.9-22.1)\end{array}$ & 1.5 & 92.5 & 1.5 & 4.5 \\
\hline Intentions to Communicate & $\begin{array}{c}40.7 \\
(32.5-48.9)\end{array}$ & 21.0 & 67.9 & 7.4 & 3.7 \\
\hline Miscellaneous Intentions & $\begin{array}{c}25.1 \\
(19.7-30.5)\end{array}$ & 11.6 & 48.8 & 12.8 & 26.8 \\
\hline Column Averages & $\begin{array}{c}24.9 \\
(20.6-29.2)\end{array}$ & 12.9 & 58.5 & 13.7 & 14.9 \\
\hline Recorders & $\begin{array}{c}27.3 \\
(24.3-30.7)\end{array}$ & 16.1 & 57.6 & 11.4 & 14.9 \\
\hline Nonrecorders & $\begin{array}{c}25.9 \\
(21.9-29.9)\end{array}$ & 6.8 & 59.3 & 15.7 & 18.2 \\
\hline
\end{tabular}

Note-Confidence intervals are given in parentheses.

ties, etc.), (3) the intention was cancelled by someone else, and (4) fulfillment of the intention was impossible due to other circumstances (e.g., bad weather, car troubles). These results are given in Table 1 for each of the six categories of plans. ${ }^{\text {I }}$ For each category, the four reasons for noncompletion sum to $100 \%$ (i.e., rows sum to $100 \%$ ). As can be seen in Table 1, the surprising result is that the participants actually overtly forgot very few of their intentions (i.e., approximately $13 \%$ in the "Column Averages" row of Table 1). Rather, reprioritization and rescheduling dominated the participants' "failures" to carry out their intentions. This dominance of reprioritization is apparent across each and every one of the six categories $\left[Q_{C M H}(15)=83.06, p<.001\right]$.

Another interesting aspect of the data summarized in Table 1 is that approximately $29 \%$ of the participants' failures were not directly under their voluntary control (i.e., cancelled and impossible as reasons given for noncompletion). Table 1 displays the percentage of reasons cited for failing to carry out one's intentions grouped by whether or not the participants were recorders or nonrecorders. Of the reasons given, an independent samples $t$ test was conducted on each of the four types of reasons (with a Bonferroni correction for multiple comparisons). On none of the four reasons did the recorders and the nonrecorders significantly differ after correcting the Type I error rate. Finally, there was a small but reliable difference in rated plan importance. Successfully completed plans were rated slightly more important during the first session $(5.9, \mathrm{CI}=5.7-6.1)$ than those that went uncompleted $(5.2, \mathrm{CI}=5.1-5.3)$. As discussed earlier, this finding is not at all surprising given that manipulations of intention importance often lead to better prospective memory (e.g., Kvavilashvili, 1992).

Attention and memory measures. Given that the recorders and the nonrecorders were similar in their overall success rate, there must be a principled reason why some people feel compelled to rely on daily planners and other related memory aids. To investigate possible underlying reasons that might stem from individual differences, Table 2 sets forth the results of the three memory measures and the three attention measures for recorders and nonrecorders separately. For each variable, performance is given as the proportion of the highest possible

Table 2

Performance on the Memory and Attention Capacity Measures as Proportions of Optimal Score for Recorders and Nonrecorders in Experiment 1

\begin{tabular}{lccccccc}
\hline & \multicolumn{3}{c}{ Memory Measures } & & \multicolumn{3}{c}{ Attention Measures } \\
\cline { 2 - 4 } \cline { 7 - 8 } & EMQ & CFQ & Free Recall & & SDMT & Ruff $2 \& 7$ & EAQ \\
\hline Recorders & .70 & .45 & .50 & .50 & .55 & .49 \\
& $(.690-.710)$ & $(.436-.464)$ & $(.474-.526)$ & & $(.490-.510)$ & $(.536-.564)$ & $(.476-.504)$ \\
Nonrecorders & .75 & .49 & .59 & & .54 & .60 & .58 \\
& $(.735-.765)$ & $(.480-.500)$ & $(.562-.618)$ & $(.530-.550)$ & $(.585-.615)$ & $(.563-.597)$ \\
\hline
\end{tabular}

Note-Confidence intervals are given in parentheses. 
score (with the CFQ reversed scored so that higher numbers reflect better performance).

As is obvious from the CIs in Table 2, the recorders and the nonrecorders performed differently on each of the EMQ, CFQ, and free recall measures of memory. The participants who did not use daily planners reported that they had better overall memory, experienced fewer failures of action and memory, and demonstrated their superior memory in the laboratory test of free recall. Results for the attention measures revealed a similar difference. The participants who did not use daily planners reported having better attentional capability as indicated by the EAQ and actually performed better than those who did use planners on the SDMT and on the $2 \& 7$ test (see the CIs in Table 2). These data indicate that the recorders said they had worse memory abilities, worse attentional capabilities, and actually performed more poorly on objective measures of attention and memory as compared with the nonrecorders. Thus, there seems to be a principled reason that some people feel compelled to carry and to use daily planners. Calendars and planners may be a compensatory device for those who believe (either rightly or wrongly) that their memory will fail them unless some external aid is used. Because this is a novel finding, a subset of these individual difference analyses was repeated again in Experiment 2.

\section{EXPERIMENT 2}

Experiment 1 examined participants' success at completing intentions they had established for themselves over the course of a week - a period that is arguably intermediate in length. The major results were as follows. First, overt forgetting of intentions was rather low, but the participants reported extensive revising, rescheduling, and changing of their plans to meet unexpected and more pressing demands. This result is not overly surprising, but it may qualify our interpretation of the research participants' performance when they failed to press a key in a laboratory event-based task (an important point that is taken up in the General Discussion section). Second, although the recorders and the nonrecorders did not differ in their total number and types of plans or in their overall success at completing their intentions, they did significantly differ on all six measures of memory and attention. The pattern of data was unambiguous: the participants who recorded their intentions believed that they had worse memory and attentional capacities, and their beliefs were borne out in more objective measures of those capacities. Third, the participants tended to complete activities where an established commitment had already been arranged, but they tended to reschedule activities that lacked this committed component (cf. Meacham, 1988).

On the basis of these findings, the goal of Experiment 2 was to explore why recorders and nonrecorders have equivalent rates of forgetting despite the recorder's perceived need for an extra memory aid and their reported habitual use of it in debriefings. One obvious candidate difference between recorders and nonrecorders is the number of times a day that they review, revise, or otherwise refresh their "list" of upcoming obligations. On the one hand, if recorders have worse memory, then perhaps they must check their daily planners and review their plans more often throughout the day. On the other hand, perhaps carrying a daily planner alleviates the need to review and to plan one's schedule as often in the course of everyday cognition. Thus, an a priori prediction is unclear concerning who reviews more often, recorders or nonrecorders. In Experiment 2, all participants were given hand-held tally counters and asked to record the number of times they actually reviewed their plans or made decisions about doing one activity from among several alternative intentions. Because approximately $60 \%$ of the participants used daily planners in Experiment 1, we were confident that enough recorders and nonrecorders could be recruited to get stable estimates of how often these two groups reviewed their plans.

Of course, the criticism exists that carrying the tally counter might cause all participants to check more often, thereby obscuring any differences between recorders and nonrecorders. A companion criticism is that carrying the tally counters affects one group differently. In terms of experimental design, this criticism is best addressed a priori by orthogonally crossing the first factor of interest (natural planner use) with a second factor of interest and examining the interaction term for significance. The second factor that was chosen required that half of the participants wear neon green wristbands as a reminder to review their intentions more frequently. Thus, the participants were asked to engage in reviewing and rehearsal of the plans they had established for the near and intermediate term every time that the wristband reminded them to do so. Hitherto, all variables manipulated in the laboratory that are known to alter retrospective remembering have been shown to similarly alter prospective remembering (see Einstein \& McDaniel, 1996, for a review; but see Vortac \& Edwards, 1995). Thus, a manipulation, such as rehearsal, that is known to improve retrospective memory should similarly improve prospective memory. Because carrying the tally counter might confound testing such a prediction, investigating the relationship between rehearsal and prospective remembering was delayed until Experiment 3, after it was confirmed that the wristband manipulation would affect the number of times a day that participants actually reviewed their plans.

\section{Method}

Participants. Eighty-two undergraduates from the University of Georgia volunteered and received course credit for their participation. The participants were tested in six groups of $10-15$ people. As in Experiment 1, each group was tested in two sessions; however, unlike before, these sessions were conducted 5 days apart. Cell sizes for the groups are provided in the note of Table 3.

Materials. Although the participants' ultimate success was not assessed in this experiment, the activity planning sheets were collected during the first session to assess whether the groups had ap- 
Table 3

Tally Counts and Capacity Measures for Experiment 2

\begin{tabular}{|c|c|c|c|c|c|c|c|c|}
\hline & \multicolumn{4}{|c|}{ Nonrecorders } & \multicolumn{4}{|c|}{ Recorders } \\
\hline & \multicolumn{2}{|c|}{ No WB } & \multicolumn{2}{|c|}{ WB } & \multicolumn{2}{|c|}{ No WB } & \multicolumn{2}{|r|}{ WB } \\
\hline & Avg. & $\mathrm{CI}$ & Avg. & $\mathrm{CI}$ & Avg. & $\mathrm{CI}$ & Avg. & $\mathrm{CI}$ \\
\hline Tally count (\#) & 40.1 & $30.6-49.6$ & 62.2 & $54.4-70.0$ & 29.3 & $22.4-36.2$ & 41.7 & $33.7-49.7$ \\
\hline Free recall $(\%)$ & 49.3 & $43.3-55.3$ & 45.4 & $38.4-52.4$ & 35.6 & $32.0-39.4$ & 39.7 & $35.7-43.7$ \\
\hline SDMT (\%) & 54.4 & $50.7-58.1$ & 53.4 & $49.2-57.6$ & 50.5 & $48.1-52.9$ & 51.3 & $48.4-54.2$ \\
\hline Ruff $2 \& 7(\%)$ & 67.7 & $63.3-72.1$ & 59.7 & $54.2-65.2$ & 56.3 & $52.8-59.8$ & 59.8 & $56.0-63.6$ \\
\hline
\end{tabular}

Note-No WB, no wristband; WB, wristband. For the nonrecorders with no wristbands, cell $N=$ 15 , and number of plans $=14.0(\mathrm{CI}=12.3-15.7)$; for the nonrecorders with wristbands, cell $N=$ 16 , and number of plans $=16.1(\mathrm{CI}=14.0-18.2)$. For the recorders with no wristbands, cell $N=25$, and number of plans $=14.3(\mathrm{Cl}=12.1-16.5)$; for the recorders with wristbands, cell $N=26$, and number of plans $=18.3(\mathrm{CI}=15.6-21.0)$.

proximately the same number of plans (as the recorders and the nonrecorders did in Experiment 1). Otherwise, all forms and measures were identical to those used in Experiment 1 . The only other difference was that the subjective capacity measures (i.e., CFQ, EMQ, and $E A Q$ ) were eliminated because they yielded the same results as the more objective assessments of attentional and memorial capacities. In addition to these materials, the participants used a tally counter to record how often they reviewed their plans over a critical 3-day period of the experiment. This hand-held mechanical device is often used for counting inventory or the number of people entering a stadium or park. Some of the participants were also required to wear a nonremovable wristband for the same period. The participants were given detailed instruction sheets to accompany the counters and the wristbands, as described next.

Procedure. The first session was always held on a Monday. After the participants documented their plans on the planning form, each was given a tally counter. Each counter had a number engraved on the side, which the participants recorded on their planning form. The participants were encouraged to click the counters for a while to become familiar with them, and they were then given detailed instruction sheets to accompany them. The instructions detailed under what conditions the counters were to be incremented. These conditions included thinking about or reviewing one's plans, as well as when decisions were made about doing one activity from among several alternative intentions. The experimenter detailed these conditions and answered any questions that arose about their prescribed use for the ensuing 3 days. The counters were to be used starting on Tuesday morning and stopping at bedtime on Thursday evening. The counter was returned during the second session, held on Friday of the same week. The number on the counter was assumed to accurately reflect the number of times the participants reviewed their upcoming plans for the rest of that day, the following day, and remainder of the week. ${ }^{2}$

Some of the participants were dismissed from this first session, and the remaining ones had the wristband affixed to their right wrist. Approximately half $(n=26)$ of the recorders and half $(n=$ 16) of the nonrecorders received wristbands. The wristbands were also accompanied by instruction sheets, detailing that the wristbands were to be used as reminders to review one's plans for the remainder of the current day, the following day, and the remainder of the week. As with the tally counter instructions, these written instructions were verbally reinforced, and questions about the prescribed reviewing and rehearsing were addressed. The participants were encouraged to "buy into" the notion that reviewing one's plans should have the beneficial outcome of improving the "amount one accomplishes over the course of the week." The participants wearing the wristbands had them removed when they handed in their tally counters. During the second session, the participants completed the retrospective, free-recall memory test. The two objective attention measures, as detailed in Experiment 1, were also administered at that time.

\section{Results and Discussion}

Providing half of each of the recorders and the nonrecorders with wristbands created an experimental design with two levels on each of two between-subjects factors (i.e., four groups of participants). Table 3 sets forth the results. As seen in the note of Table 3 , the recorders and the nonrecorders did not differ in their total number of plans. However, the participants randomly chosen to wear wristbands did have a slightly greater number of plans. This average of three extra plans for those who wore wristbands cannot account for the increased rehearsal of their plans, as described next. Table 3 specifies the total number of times the participants reviewed their plans over the course of the 3 days from Tuesday morning to Thursday evening (i.e., the tally count). Two effects are noteworthy. First, the participants who were given the wristbands reviewed their plans almost $50 \%$ more frequently than did those without wristbands. This outcome was anticipated if the wristbands had successfully served as a reminder to rehearse and review one's upcoming obligations. Second, the more interesting outcome was that the recorders generally thought about their intentions much less frequently than did the nonrecorders. For example, the recorders without wristbands reviewed their plans about 10.8 times less often (i.e., about 3 times a day less often) than did their nonrecorder counterparts without wristbands, even though both groups had an equal number of plans. Perhaps carrying a daily planner obviates the need to check one's obligations as frequently in order to successfully accomplish upcoming activities. Or perhaps carrying the planner instills a level of confidence about meeting one's obligations such that one reviews one's intentions less frequently.

Whatever the specific reason, people who use a memory aid to accomplish their planned activities think about those commitments less frequently, as objectively measured in this experiment. What this experiment failed to address is how people use those aids. Clearly, they do not 
write down everything in their daily planners. Perhaps they write down only a fraction of their intentions. An analysis of that sort is beyond the scope of this article. To foreshadow, however, 43 participants in Experiment 3 had their daily planners taken away for a week. In a casual analysis of those daily planners (with the participants' permission), we found that people do use them quite a bit. Generally, almost everyone had at least one activity per day, and some participants used them both as a prospective device and as a retrospective diary. Although exactly how they are used is unknown, the results of Experiment 2 argue that recorders think about their intentions and obligations less often. Importantly, no difference in tally counter use was observed as a function of the treatment combination of the wristband manipulation and the participants' use of a planner $[F(1,78)=1.29, p>.2]$.

The objective measures of attention and memory were administered, as in Experiment 1. As seen in the CIs in Table 3, the recorders again had poorer retrospective memory than did the nonrecorders. They also had worse attentional capacity as measured by the $2 \& 7$ test; however, the results of the SDMT, having the predicted direction from Experiment 1, would only be "marginal" by conventional significance testing. We found that, across two experiments, with over 200 participants, people who record their intentions say they have worse attention and memory capacities; this fact was borne out again in Experiment 2 by objective tests of those capacities. One explanation for this finding, as discussed earlier, is simply that daily planners are used as a compensatory strategy by people who believe that they will not successfully remember activities unless they use this particular memory aid. For others (about $40 \%$ in Experiment 2), mentally reviewing one's obligations more frequently probably served them equally well in accomplishing the tasks they established for themselves. One explanation for the difference in frequency of reviewing that was ruled out by Experiment 1 is that recorders and nonrecorders have qualitatively different types of plans and commitments; they do not (see Experiment 1 and Table 1).

\section{EXPERIMENT 3}

Experiment 1 demonstrated that people's prospective memory performance was equivalent regardless of one's status as a recorder or a nonrecorder. Experiment 2 demonstrated, however, that these two groups differed on the important variable of how often they reviewed and revised their planned activities. Together, the two experiments suggest that there may be important differences in how the two groups achieve equivalent overall success. The nonrecorders may have established a routine of frequently thinking about their intentions and obligations, perhaps because they knew how fallible human memory could be. The recorders, on the other hand, may have consulted their daily planners less frequently because of the permanence associated with writing obligations down. In Experiment 3, participants who were recorders were transformed into nonrecorders, and vice versa. This manipulation was intended to assess how nonrecorders would react to newly keeping a daily planner, and how recorders would react to having their memory aid taken away. One obvious prediction is that recorders who have their daily planners taken away should exhibit more frequent failures of prospective memory. In contrast, giving nonrecorders a daily planner may not result in any benefit, because they have grown accustomed to mentally reviewing their plans more frequently.

Another goal of Experiment 3 was to assess whether the rehearsal manipulation of wearing a wristband would affect actual everyday prospective memory performance. As reflected in significantly greater numbers recorded on the tally counters in Experiment 2, wearing the wristband increased the rehearsal and reviewing of the participants' plans. Theoretically, greater rehearsal of one's intentions should be reflected in fewer overtly forgotten plans or in greater numbers of reprioritized plans, as assessed by the paradigm used in Experiment 1. Thus, Experiment 3 replicated the procedure of Experiment 1 with two additional factors besides the planner-use variable. Orthogonally crossing planner use with additional rehearsal by wearing a wristband and either provision of a planner or removal of one's own planner yielded a $2 \times 2 \times 2$ entirely between-subjects factorial design.

\section{Method}

Participants. One hundred eighty-eight people volunteered in return for partial fulfillment of a research requirement. None had participated in Experiment 1 or Experiment 2. As in Experiment 1, the participants were tested in two sessions spaced 7 days apart. Cell sizes provided in the note of Table 4 averaged approximately 24 people.

Procedure. Aspects of the procedure were identical to Experiments 1 and 2. During the first session, the participants filled out the activity planning sheets. Half of the recorders had their daily planners taken away, and half of the nonrecorders were provided with daily planners. The daily planners provided to the participants were the seven $3.5 \times 6$ in. sheets from a standard desk calendar, bound, for the appropriate week in which the study was conducted. The participants were encouraged to use the planner, and they were told that the daily planners would be collected at the end of the study for analysis. To get these new users accustomed to using their planner, they were asked to transfer their plans from the planning activity sheets to the newly provided planners. With the participants now subdivided into four groups, they were further subdivided. Half of each group was dismissed, with the remaining participants being trained on the prescribed regimen of reviewing their intended activities whenever the wristband reminded them to do so. Nonremovable neon green wristbands were affixed to the right wrist before leaving this first session. During the second session, 1 week later, everyone documented which of their plans they had completed. If they had been unsuccessful, then they provided a reason for why the intention went unfulfilled. Each participant with a wristband had it removed during this second session. Thus, unlike in Experiment 2 in which there was a critical 3-day rehearsal manipulation, the rehearsal manipulation lasted the entire 7 days in Experiment 3. Because of the large number of participants in Experiment 3, the memory and attention capacity measures were not administered 
Table 4

Percentages of Uncompleted Plans by Category in Experiment 3

\begin{tabular}{|c|c|c|c|c|c|c|c|c|c|c|c|c|c|c|c|c|}
\hline & \multicolumn{8}{|c|}{ Natural Nonrecorders } & \multicolumn{8}{|c|}{ Natural Recorders } \\
\hline & \multicolumn{4}{|c|}{ No Planner } & \multicolumn{4}{|c|}{ Planner } & \multicolumn{4}{|c|}{ No Planner } & \multicolumn{4}{|c|}{ Planner } \\
\hline & \multicolumn{2}{|c|}{ No WB } & \multicolumn{2}{|c|}{ WB } & \multicolumn{2}{|c|}{ No WB } & \multicolumn{2}{|c|}{ WB } & \multicolumn{2}{|c|}{ No WB } & \multicolumn{2}{|c|}{ WB } & \multicolumn{2}{|c|}{ No WB } & \multicolumn{2}{|c|}{ WB } \\
\hline & Avg. & $S D$ & Avg. & $S D$ & Avg. & $S D$ & Avg. & $S D$ & Avg. & $S D$ & Avg. & $S D$ & Avg. & $S D$ & Avg. & $S D$ \\
\hline & 23.6 & 14.8 & 26.9 & 13.1 & 23.7 & 14.1 & 24.1 & 11.6 & 20.8 & 14.5 & 23.3 & 17.3 & 24.2 & 14.4 & 23.3 & 13.5 \\
\hline & 19.8 & 20.5 & 14.1 & 15.6 & 11.1 & 18.4 & 4.9 & 11.1 & 16.2 & 27.0 & 23.6 & 25.1 & 6.3 & 10.5 & 14.9 & 24.3 \\
\hline ritized & 59.9 & 31.1 & 56.4 & 26.1 & 64.3 & 32.3 & 68.6 & 26.8 & 71.9 & 31.0 & 62.4 & 28.4 & 74.0 & 29.1 & 60.2 & 32.1 \\
\hline Cance & 2.7 & 8.5 & 10.5 & 14.9 & 8.9 & 14.0 & 13.9 & 22.9 & 7.7 & 16.3 & 6.6 & 11.8 & 5.1 & 10.0 & 7.5 & 21.4 \\
\hline Impo & 17.6 & 31.0 & 19.0 & 21.9 & 15.6 & 22.4 & 12.7 & 16.1 & 4.2 & 8.4 & 7.4 & 17.1 & 12.4 & 23.0 & 17.3 & 25.7 \\
\hline Extrapolated tally & 93.5 & & 145.1 & & & & & & & & & & 68.4 & & 97.3 & \\
\hline
\end{tabular}

Note-No WB, no wristband; WB, wristband. For the natural nonrecorders with no planners and no wristbands, cell $N=$ 20 , and number of plans $=15.9$; for the natural nonrecorders with no planners and with wristbands, cell $N=24$, and number of plans $=17.1$. For the natural nonrecorders with planners and no wristbands, cell $N=24$, and number of plans $=16.6$; for the natural nonrecorders with planners and wristbands, cell $N=23$, and number of plans $=16.6$. For the natural recorders with no planners and no wristbands, cell $N=21$, and number of plans $=16.2$; for the natural recorders with no planners and with wristbands, cell $N=22$, and number of plans $=15.7$. For the natural recorders with planners and no wristbands, cell $N=28$, and number of plans $=17.2$; for the natural recorders with planners and wristbands, cell $N=26$, and number of plans $=20.3$.

\section{Results and Discussion}

The results are set forth in Table 4. For purposes of comparison, the leftmost two conditions (no planners with and without wristbands) and the rightmost two conditions of Table 4 (planners with and without wristbands) are the four cells that replicate the conditions tested in Experiment 2.3 As can be seen in the note of Table 4, cell sizes were fairly uniform ranging from 20 to 28 participants. In addition, the average number of plans was fairly uniform, and it did not differ as a function of any of the three factors of interest [all $F_{\mathrm{s}}(1,180)<2.5$, n.s.]. For those participants who failed to complete at least one activity, the overall noncompletion rate did not differ by the conditions of interest, the largest $F$ ratio among the main effects and interactions being 0.65 , n.s. Recall that overt forgetting and reprioritization are the only two reasons for noncompletion that are under a person's control. In terms of overt forgetting, two outcomes are noteworthy.

First, the participants who carried a daily planner forgot significantly fewer commitments $[F(1,167)=9.56$, $p<.01]$. Interestingly, this factor did not significantly interact with either of the other factors. Therefore, natural recorders and natural nonrecorders both benefited from having the memory aid of keeping a daily planner. Although providing a planner to natural nonrecorders need not have improved their performance because they review their intentions more often anyway, it nevertheless did improve their prospective memory, and they overtly forgot fewer commitments $[t(86)=2.49, p<.02]$. This main effect of carrying a planner also suggests that the participants who had their planners taken away forgot significantly more intended activities than they otherwise would have $[t(85)=2.0, p<.05]$.

Second, there was a significant interaction between natural recorder status and the wristband rehearsal manipulation $[F(1,167)=5.67, p<.02]$. This interaction concerning overt forgetting is both interesting and easily interpretable. For natural nonrecorders, the wristband re- hearsal manipulation acted to reduce overt forgetting. In contrast, for natural recorders, the wristband rehearsal manipulation increased overt forgetting. Momentarily extrapolating the 3-day tally count collected in Experiment 2 to 7 days yields the results in the last row of Table 4 (speculation on the inner columns of this table would be premature). Increasing the number of times natural nonrecorders reviewed their intentions from approximately 94-145 times during the week dramatically reduced overt forgetting. In contrast, increasing natural recorders from reviewing 68-97 times during the week increased overt forgetting. One interpretation of this pattern of results is that natural nonrecorders already mentally review their plans to a greater degree (cf. Experiment 2), and any increment extends (or is more of) the cognitive processing that they are already naturally engaging in order to fulfill their intentions. For natural recorders, they do not normally mentally rehearse or review their intentions as often, and doing so somehow interferes with accomplishing the activities they had intended. As mentioned earlier, without knowing exactly how people interact with their daily planners, it is difficult to precisely pinpoint the cognitive mechanism underlying this result for the recorders. Importantly, rehearsal does not uniformly improve prospective memory, as years of research on retrospective memory would have predicted (cf. Vortac \& Edwards, 1995). Rather, rehearsal interacts with status as a recorder or a nonrecorder and, therefore, perhaps with the underlying capacity differences in attention and memory that were found in Experiments 1 and 2.

There was no evidence for any other interesting results in the data for overtly forgotten or for reprioritized plans. The fact that natural recorder status was not predictive of either the overall noncompletion rate or the proportion of overtly forgotten plans wholly replicated the results of Experiment 1. Differences only emerged as a function of the planner and rehearsal manipulations, as just described. We now consider what the three experiments, 
and this paradigm, might have to say about how people complete the intentions they establish for themselves, as compared with more controlled laboratory observations of prospective remembering.

\section{GENERAL DISCUSSION}

In these experiments, we studied prospective memory by examining individual differences in both cognitive capacities and strategic approaches to remembering to carry out one's everyday plans. By relaxing laboratory control in favor of ecological validity, the three experiments revealed a number of interesting findings about how people might remember to carry out their intentions. First, the participants in Experiment 1 remembered to fulfill intentions about prearranged appointments and those that involved a commitment to another person (cf. Meacham, 1988). They were, however, less diligent in fulfilling intentions to arrange appointments, to take or to return things, and so on. Second, contrary to popular belief, we found, in Experiments 1 and 3, that people overtly forget very few of their plans; the participants reported that they consciously reprioritized intentions as current demands dictated and as other opportunities and obligations arose. Third, as compared with the nonrecorders, the recorders in Experiments 1 and 3 neither had nor did they complete larger numbers of intentions. The means by which recorders and nonrecorders accomplish their prospective remembering, however, may differ substantially, as follows. Fourth, the natural recorders in Experiments 1 and 2 showed small (but reliable and consistent) deficits in both attentional and memorial capacities, as compared with the nonrecorders. Fifth, recorders in Experiment 2 thought less frequently about their obligations over a 3-day period than did the nonrecorders. Sixth, mental rehearsal of one's obligations in Experiment 3 appeared to be beneficial for the nonrecorders who normally commit their obligations to memory anyway but was detrimental for the recorders who normally write down those same obligations on paper. Seventh, and finally, the results of Experiment 3 suggest that, although recorders might realize the benefit of a written record of things to be accomplished (perhaps as a consequence of having poorer memories), nonrecorders can benefit by changing their strategies to include writing things down. We now consider the implications of these findings for theories of prospective memory.

Unfortunately, comprehensive theories do not exist for how people contend with the intentions they establish for themselves. The reason that no existing theory captures a very broad range of phenomena in prospective remembering may reflect the fact that "prospective memory" is supported by many facets of cognition, including attention, planning, reprioritizing, monitoring, and retrospective memory, to name just a few. By this view, prospective remembering clearly relies on a multidimensional set of cognitive processes. For example, prospective perfor- mance outside the laboratory depends on metaknowledge (cf. Dobbs \& Reeves, 1996). That is, people know how good or how poor their memories are, what their level of motivation is, and what the completion of an intention requires. As a consequence, people take steps to adopt strategies that will compensate for their own shortcomings. In Experiments 1 and 3, the participants who used daily planners did so for a reason. They probably believe that without such aids, they would likely accomplish significantly fewer of their intentions. Metamemory for their poorer attentional and memorial capacities may have caused them to use this compensatory strategy for completing more of their intentions. Interestingly, the deficit that the recorders showed relative to the nonrecorders was not isolated to either the prospective or the retrospective components of prospective memory (momentarily adopting that dichotomy). Rather, the recorders displayed deficits in both components, if one assumes that prospective memory is supported by attentional capacities (see Marsh \& Hicks, 1998, for a discussion of how prospective memory is supported by attentional mechanisms).

Granted, the evidence for the role of attention is not very strong in Experiments 1 and 2. However, combined with other differences between recorders and nonrecorders, such as how frequently their intentions are reviewed, a picture begins to emerge regarding how one cognitive resource (e.g., good retrospective memory) augments another (e.g., more frequent monitoring) for nonrecorders that enables them to achieve the equivalent prospective memory performance of, say, keeping a written daily planner. The choice of examining memorial and attentional capacities was made solely for parsimony, and it neglects many other important variables that are not well represented in Experiments 1-3. For example, in Experiments 1 and 3 most "failed" intentions were a result of reprioritization and overt decisions on the part of the participants to perform other tasks rather than what they had intended originally. How or under what circumstances those decisions were made was not captured in Experiments 1-3. In addition, the catalogue of the participants' intentions was frozen when they left the first session.

Thus, exactly how many new intentions were established (and accomplished) through reprioritization is unknown. Although reprioritization might have dominated the reasons given for noncompletion because of the rationalization effects of hindsight, that criticism does not explain (1) why the participants often wrote down what the specific activities were that displaced the intended activities and (2) their willingness to tell us about many of their overt failures (approaching 13\%). This basic issue is what experimentalists have labeled item selection effects. Because the participants chose for themselves which plans they would and would not complete, those assigned to a given condition in Experiments 1 and 3 were not uniform in any way. While sensitive to this issue, we note that the behavior under criticism is exactly the behavior that we wanted to investigate. The study of prospective memory 
outside the laboratory is the study of item selection effects as they relate to participant-established intentions, and not experimenter-provided intentions.

Better metrics of planning and reprioritization, however, than those used in Experiments 1 and 3 could prove important in investigations of participant-established intentions. The results of such metrics will eventually have to be incorporated into theories of prospective memory if those theories are to have significant explanatory power for prospective memory that occurs outside of the laboratory. When a participant fails to press a key in a laboratorybased experiment of prospective remembering, that omission is rightfully labeled "overt forgetting." In moving from the realm of the short-term intentions studied in the laboratory to longer term intentions that people establish for themselves, the results of Experiments 1 and 3 suggest some caution in labeling unfulfilled intentions as prospective memory "failures" (cf. Ellis, 1996, and Roediger, 1996, who drew the same conclusion). They may not be failures at all but, rather, conscious decisions on the part of people to postpone or to cancel a previously established intention (cf. Ellis, 1996).

Rabbit (1996) recently observed that the general laboratory approach to studying prospective memory involved empirical studies that were "planless." By this, he meant that there exists a larger and richer role for other cognitive components that support completing the plans that people establish for themselves. The paradigm used in Experiments 1 and 3 placed special emphasis on variables that have not yet been examined in the empirical study of how people contend with their everyday intentions. In doing so, several factors quite unrelated to retrospective memory were found to be important (viz., attention, metamemory, and planning as it relates to reprioritization of old and newly established intentions). Although somewhat descriptive, by investigating individual differences in attention and the strategic use of memory aids, a glimpse into the complex cognitive interactions of the multiple components of prospective remembering was obtained (e.g., Dobbs \& Reeves, 1996; Ellis, 1996). Although laboratory tasks will continue to dominate future experimentation on prospective memory, the current paradigm was used to manipulate factors thought to be intuitively important to prospective remembering. For example, the wristband manipulation of rehearsal in Experiment 3 altered the reviewing and reprioritizing processes that the participants naturally undertook. That same manipulation demonstrated qualitatively different results depending on whether memory aids, such as daily planners, were taken away as compared with when they were provided to people who did not normally use them. Besides the descriptive and empirical manipulations reported in this article, we note more generally, when an ecologically complex behavior is being investigated, studying that behavior nearer to in vivo conditions can often yield important insights into that behavior. We believe that, for those interested in studying prospective memory, our seven basic findings should provide solid points of departure for future development with investigations both outside and inside the laboratory (cf. Marsh \& Hicks, 1998).

\section{REFERENCES}

BEAL, C. R. (1988). The development of prospective memory skills. In M. M. Gruneberg, P. E. Morris, \& R. N. Sykes (Eds.), Practical aspects of memory: Current research and issues (Vol. 1, pp. 366-370). Chichester, U.K.: Wiley.

Broadbent, D. E., Cooper, P. F., Fitzgerald, P., \& Parkes, K. R. (1982). The Cognitive Failures Questionnaire (CFQ) and its correlates. British Journal of Clinical Psychology, 21, 1-16.

CraIK, F. I. M. (1986). A functional account of age differences in memory. In F. Klix \& H. Hagendorf(Eds.), Human memory and cognitive capabilities: Mechanisms and performances (pp. 409-422). Amsterdam: Elsevier, North-Holland.

DoBbs, A. R., \& ReEves, M. B. (1996). Prospective memory: More than memory. In M. Brandimonte, G. O. Einstein, \& M. A. McDaniel (Eds.), Prospective memory: Theory and applications (pp. 199-225). Hillsdale, NJ: Erlbaum.

DoBbs, A. R., \& RulE, B. G. (1987). Prospective memory and selfreports of memory abilities in older adults. Canadian Journal of Psychology, 41, 209-222.

Einstein, G. O., \& McDaniel, M. A. (1990). Normal aging and prospective memory. Journal of Experimental Psychology: Learning, Memory, \& Cognition, 16, 717-726.

EinsteIn, G. O., \& MCDANIEL, M. A. (1996). Retrieval processes in prospective memory: Theoretical approaches and some new empirical findings. In M. Brandimonte, G. O. Einstein, \& M. A. McDaniel (Eds.), Prospective memory: Theory and applications (pp. 115-141). Hillsdale, NJ: Erlbaum.

Einstein, G. O., MCDaniel, M. A., Richardson, S. L., Guynn, M. J., \& CunFer, A. R. (1995). Aging and prospective memory: Examining the influences of self-initiated retrieval processes. Journal of Experimental Psychology: Learning, Memory, \& Cognition, 21, 996-1007.

ELLIS, J. A. (1996). Prospective memory or the realization of delayed intentions: A conceptual framework for research. In M. Brandimonte, G. O. Einstein, \& M. A. McDaniel (Eds.), Prospective memory: Theory and applications (pp. 1-22). Hillsdale, NJ: Erlbaum.

ELLIS, J. A., \& NiмMO-SмITH, I. (1993). Recollecting naturally-occurring intentions: A study of cognitive and affective factors. Memory, $\mathbf{1}$, $107-126$

HARRIS, J. E. (1980). Memory aids people use: Two interview studies. Memory \& Cognition, 8, 31-38.

KVAVILASH VILI, L. (1987). Remembering intention as a distinct form of memory. British Journal of Psychology, 78, 507-518.

KVAVILASH VILI, L. (1992). Remembering intentions: A critical review of existing experimental paradigms. Applied Cognitive Psychology, 6, 507-524.

LofTUS, E. F. (1971). Memory for intentions: The effect of presence of a cue and interpolated activity. Psychonomic Science, 23, 31 5-316.

Marsh, R. L., \& Hicks, J. L. (1998). Event-based prospective memory and executive control of working memory. Journal of Experimental Psychology: Learning, Memory, \& Cognition, 24, 336-349.

MARTIN, M. (1986). Aging and patterns of change in everyday memory and cognition. Human Learning, 5, 63-74

Mayhorn, C. B., Park, D. C., Morrell, R. W., \& Marsh, R. L. (1995, August). The effects of cognitive and psychosocial factors on prospective memory tasks performed in laboratory and naturalistic settings. Paper presented at the 103rd Annual Meeting of the American Psychological Association, New York.

MAYLOR, E. A. (1990). Age and prospective memory. Quarterly Journal of Experimental Psychology, 42A, 471-493. 
Meacham, J. A. (1988). Interpersonal relations and prospective remembering. In M. M. Gruneberg, P. E. Morris, \& R. N. Sykes (Eds.), Practical aspects of memory: Current research and issues (Vol. 1, pp. 354-359). Chichester, U.K.: Wiley.

MeAChaM, J. A., \& KuShner, S. (1980). Anxiety, prospective remembering and performance of planned actions. Journal of General Psychology, 103, 203-209.

Meacham, J. A., \& Singer, J. (1977). Incentive effects in prospective remembering. Journal of Psychology, 97, 191-197.

Moscovitch, M. (1982). A neuropsychological approach to perception and memory in normal and pathological aging. In F. I. M. Craik \& $\mathrm{S}$. Trehub (Eds.), Advances in the study of communication and affect. Vol. 8. Aging and cognitive processes (pp. 55-78). New York: Plenum.

Park, D. C., Smith, A. D., \& Cavanaugh, J. C. (1990). Metamemories of memory researchers. Memory \& Cognition, 18, 321-327.

RABBIT, P. (1996). Why are studies of "prospective memory" planless? In M. Brandimonte, G. O. Einstein, \& M. A. McDaniel (Eds.), Prospective memory: Theory and applications (pp. 239-248). Hillsdale, NJ: Erlbaum.

RoEDIGER, H. R., III (1996). Prospective memory and episodic memory. In M. Brandimonte, G. O. Einstein, \& M. A. McDaniel (Eds.), Prospective memory: Theory and applications (pp. 149-155). Hillsdale, NJ: Erlbaum.

Ruff, R. M., Niemann, H., Allen, C. C., Farrow, C. E., \& Wylie, T. (1986). Automatic detection vs. controlled search: A paper-and-pencil approach. Perceptual \& Motor Skills, 62, 407-416.

SmITH, A. (1968). The Symbol Digit Modalities Test: A neuropsychologic test for economic screening of learning and other cerebral disorders. Learning Disorders, 3, 83-91.

VorTaC, O. U., \& EDWARdS, M. B. (1995). Functions of external cues in prospective memory. Memory, 3, 201-219.

WiLKINS, A. J., \& BADDELEY, A. D. (1978). Remembering to recall in everyday life: An approach to absent-mindedness. In M. M. Gruneberg, P. E. Morris, \& R. N. Sykes (Eds.), Practical aspects of memory (pp. 27-34). London: Academic Press.

\section{NOTES}

1. Given the classification of the participants' intentions into six categories and reasons for failure into four categories, the 24 resulting combinations could not be analyzed by a within-subjects analysis of variance (because each person was not observed in each cell). The differences among the means in Table 1 were assessed based on the overall frequency using nonparametric statistics. Because the participants contributed to more than one category by reason cell of Table 1, standard nonparametric statistics (i.e., chi-square) do not account for the dependence of observations among the cells. Therefore, a Cochran-MantelHaenszel test of association was conducted on the frequencies that generated the averages in Table 1 in order to account for this dependence.

2. Remembering to increment the tally counter requires a form of prospective memory. Given that the recorders and the nonrecorders did not differ in their prospective memory performance, we assumed any failures of prospective memory to increment the counters should be equivalent across tasks and conditions.

3. Although the data could be classified by the six categories of plans as in Experiment 1, rather than laboriously repeat the presentation of that analysis, we note that this analysis did not yield any additional insights beyond those reported in Experiment 1 . Note that, with the number of conditions in Experiment 3, such a table of results would have a minimum of 192 percentages.

(Manuscript received August 21, 1996; revision accepted for publication February 27, 1997.) 